Euskal ikerketen aldizkaria | Revue d'études basques |

Revista de estudios vascos | Basque studies review

$17 \mid 2013$

Numéro XVII

\title{
Georges Hérelle et le théâtre basque
}

\section{Eric Dicharry}

\section{OpenEdition}

Journals

Édition électronique

URL : https://journals.openedition.org/lapurdum/2403

DOI : 10.4000/lapurdum.2403

ISSN : 1965-0655

Éditeur

IKER

Édition imprimée

Date de publication : 1 octobre 2013

Pagination : 31-35

ISBN : 978-2-86781-409-9

ISSN : $1273-3830$

\section{Référence électronique}

Eric Dicharry, « Georges Hérelle et le théâtre basque », Lapurdum [En ligne], 17| 2013, mis en ligne le 15 novembre 2015, consulté le 23 juillet 2021. URL : http://journals.openedition.org/lapurdum/2403 ; DOI : https://doi.org/10.4000/lapurdum.2403 


\title{
Georges Hérelle et le théâtre basque
}

\author{
Eric DICHARRY
}

\section{Biographie}

Georges Hérelle est né à Pougy-sur-Aube le 27 Août 1848 d'un père Picard et d'une mère Champenoise. Après un parcours scolaire brillant au collège de Troyes où son père était professeur, il part terminer ses études à Paris où il fut trois fois lauréat du Concours général au Lycée Louis-le-Grand. Licencié ès lettres, il devient en 1871 professeur de philosophie aux collèges de Dieppe et de Vitry-le-François, puis aux lycées d'Evreux et de Cherbourg. En 1896 il obtient une réponse favorable à sa demande de mutation pour Bayonne. Il s'installe dès lors au Pays Basque et poursuit son enseignement en philosophie jusqu'à l'année de sa retraite en 1903. Il consacre alors son temps à l'étude du théâtre souletin en assistant à partir de 1899 à de nombreuses représentations. Cette même année il participe à sa première pastorale dans le village de Haux. Il s'intéresse aussi aux mascarades et prend part de 1901 à 1914 à cinq carnavals. Il publie de nombreux articles et ouvrages sur le théâtre en Soule, en France et en Europe. Il décède le 15 décembre 1935 à Bayonne

Le fonds ethnographique déposé à la Bibliothèque Municipale de Bayonne

\section{Description du fonds.}

Il est composé

1) de manuscrits de pastorales souletines et de traductions dont l'auteur avec la collaboration de souletins a fait l'acquisition pour le compte de la Bibliothèque Nationale1. Il a remis ainsi 14 manuscrits de pastorales à la bibliothèque municipale de 
Bayonne (Saint Claudiens ou Mustapha, Alexandre, Charlemagne, Sainte Geneviève, Saint Jacques, Saint Jean, Saint Louis, Richard sans peur, Sainte Margerite, Napoléon, Astyage, Nabuchodonosor, Saint Roch, Clovis ). Il a de plus réalisé une analyse et une traduction des manuscrits basques de la Bibliothèque de Bordeaux avec l'aide de Léopold Irigaray. Il traduit des prologues et des épilogues et analyse plusieurs pièces. Il réalise la traduction complète de Hélène de Constantinople à partir de la traduction anglaise de Dogson. Léopold Irigaray traduit la pastorale Clovis et Hérelle donne l'épilogue de Jeanne d'Arc en basque. Le collaborateur souletin de Hérelle traduit de plus le prologue et l'épilogue de Napoléon Empereur.

2) D'analyses et de traductions partielles de farces charivariques qu'il retrouve à la Bibliothèque de Bordeaux. Léopold Irigaray traduit Pansart et Hérelle analyse Chiveroua et Marceline, Mehalçu et Vénus, Saturne et Vénus, Jouanic Hobe et Arlaïta, Bala et Vilota, Petitun et Petikhuni, Dorimène et le prince Osman, Malkus et Malkulina, Bacchus, Pierrot et Charrot.

3) De documents divers concernant le théâtre rural français et européen.

4) De notes sur le théâtre basque, d'analyses de pastorales, d'informations concernant les auteurs, les instituteurs, notes sur les cavalcades, charivaris, farces charivariques, mascarades.

5) De sept cartons contenant une correspondance relative aux pastorales.

La correspondance de Hérelle concernant les pastorales permettent d'accéder à l'atelier du chercheur. Elle permet de comprendre de quelle manière l'auteur à procédé pour composer ses articles et ouvrages consacrés au théâtre basque. Il s'informe auprès de bibliothécaires, archivistes, professeurs à l'université, hommes de lettres, en France mais aussi à l'étranger, en Italie, en Espagne, en Suisse. Il soumet ses hypothèses sur la versification à Louis Havet, professeur au collège de France. Il reçoit des lettres de Vinson, Webster, Julio de Urquijo qui l'ont tous lus et le félicite de ses travaux sur le théâtre basque. De nombreuses lettres rappellent sa collaboration à la revue internationale des études basques dès sa création en 1906. Il publie dans cette revue ses recherches sur les mascarades (1914-1923) et sur les charivaris nocturnes (1921). La correspondance avec Honoré Champion expriment une entente cordiale entre l'auteur et son éditeur qui publiera l'ensemble de son œuvre sur le théâtre basque (1923, 1925, 1926, 1928). Il participe de plus à partir de 1911 à la Société des Sciences, Lettres et Arts de Bayonne dont il devient le président d'honneur. Il entretient avec les instituteurs de pastorales une relation privilégiée. Ils l'informent sur les dates des représentations des pastorales et des mascarades. Ager (instituteur de pastorales) lui demande conseil pour la mise en scène. Un autre comme Heguiaphal en contrepartie de l'envoi de manuscrits (il a vendu en tout quinze manuscrits pour la somme de 950frs) désire obtenir des pièces de pastorales pour les jouer (Heguiaphal lui demande ainsi les cahiers de celles de Saint Roch, Clovis, Saint Louis). Hérelle a réalisé un questionnaire sur les pastorales (Pays Basque et Béarn) et sur les mascarades qui se trouve dans son cahier de notes de 1917. Il s'informe sur le budget, les recettes, la location des costumes, demande une description des représentations théâtrales, une localisation et une analyse des langues usitées, une liste des titres que le correspondant a eu entre les mains, des informations sur les pièces comiques, sur les pastorales en français en Béarn, sur l'influence du théâtre basque sur les pastorales en Béarn et vice versa. L'enquête déborde du cadre basque pour s'intéresser au théâtre rural en Béarn, en France et en Europe. Pour ce faire l'auteur utilise les réponses à son questionnaire. En Soule, il participe aussi aux représentations ce qui initie 
une démarche ethnographique qu'il associe à un recherche en "chambre" par la consultation de nombreux ouvrages.

Pendant plus de vingt ans Hérelle se sera passionné pour le théâtre basque et en particulier souletin. Cet homme qui vénérait la nature et les bêtes s'était épris de cette petite province basque au point de désirer acheter une maison à Larrau. Les dix volumes de notes sur les pastorales basques contiennent les sources des travaux que l'auteur publiera sur le théâtre souletin de 1908 à 1926. Les questionnaires illustrent de quelle manière ces recherches dépassent le simple cadre des pastorales pour s'intéresser à l'ensemble de la littérature orale, les mascarades, les txikito, les asto laster, les tragi-comédies de carnaval. Non bascophone, c'est grâce à Léopold Irigaray, son traducteur et informateur souletin qu'il accède au sens des textes. Sa démarche consiste à recueillir des manuscrits soit en les faisant acheter par Irigaray, soit en se les procurant lui même en les payant au propriétaire, soit par des dons de particuliers. La majorité du fonds Hérelle est constitué des manuscrits obtenus auprès de Heguiaphal (quinze au total pour une somme de 500 francs), instituteur de pastorales. Il collecte les cahiers, les décrit, fournit des informations sur le nom des acteurs, les costumes, le déroulement, la place des personnages, l'auteur du manuscrit, le propriétaire. Les traductions de prologues et d'épilogues sont réalisées par Irigaray. Pour ce qui est des tragicomédies de carnaval, il procède de manière identique. Sa participation à cinq mascarades lui permet la rédaction de deux articles qui décrivent le rite en présentant les personnages, le cortège. De 1899 à 1914, il assiste à dix pastorales sur les trente quatre données en Soule. Hérelle est incontournable pour celui qui traite du théâtre basque. Il est cité par la plupart des chercheurs, Garamendi Azcorra, Larrinoa, Oiharzabal, Haritschelhar. Ils reconnaissent ses qualités d'ethnographe mais aussi d'historien du théâtre basque.

Il recense et compulse les 207 manuscrits existants parmi lesquels 102 appartenaient à des particuliers et 105 à des dépôts publics en vue de la réalisation d'une histoire du répertoire qui lui servira de base pour sa théorie sur l'origine des pastorales selon laquelle ce théâtre dérive des mystères français et a été importé vers la fin du XVème siècle dans la Soule, lorsque les représentations de mystères faisaient fureur dans toute la France. D'autres théories émisent depuis les font elles remonter au XVIIIème siècle et ce en raison de la date des manuscrits. Les thèmes abordés par Hérelle sont repris par les chercheurs en sciences sociales et les anthropologues de la culture basque ce qui témoigne aussi d'un regain d'intérêt des chercheurs vis à vis de la littérature populaire. Chaque genre littéraire devient l'objet de publications. Mozos, Urkizu et Peillen s'intéressent aux farces charivariques. Oiharzabal et Inchauspe choisissent les pastorales comme sujet de leur thèse de doctorat.

Kepa Fernandez de Larrinoa réalise un terrain en octobre 1991 et septembre 1992 et analyse les mascarades dans le cadre d'un stage postdoctoral au centre du département de la politique scientifique du gouvernement basque. Le colloque organisé par la société d'études basques, Eusko Ikaskuntza qui s'est tenu les 13 et 14 Mai 1998 à la faculté de Bayonne qui regroupait la plupart des chercheurs spécialisés sur le théâtre populaire basque illustre encore une fois la place de ce thème initié voilà près d'un siècle par Hérelle et qui demeure essentiel dans les recherches en sciences sociales contemporaines. Ses descriptions des mascarades permettent aujourd'hui de mener une étude comparative concernant les modifications des personnages du cortège, des transformations de l'usage des langues théâtrales en fonction d'un contexte de crise où la langue basque dominée par le français trouve dans le rite un mode d'expression d'une affirmation identitaire. 
Les Souletins ont retenu de Hérelle son jugement pessimiste qui prévoyait la mort des pastorales. Ces propos sont bien souvent mis en avant et participent à leur fierté, comme si chaque représentation théâtrale signifiait la condamnation de la parole savante par l'action populaire. Le dire devient dans un contexte de diglossie symbole de vie. La souletinisation des énoncés des Noirs (personnages des mascarades qui ont accès à la parole) et la basquisation des sujets des pastorales illustrent les modifications qui ont permis aux rites de se perpétuer et le repli de la langue dans des formes d'expression littéraires léguées par la tradition et qui participent désormais à la dynamique culturelle basque. A l'extraordinaire variété des genres littéraires qui existaient à l'époque de Hérelle succède quelques rescapés comme les pastorales et les mascarades ce qui va dans le sens des analyses de l'auteur qui mettait l'accent sur la disparition prochaine de certains genres. Son jugement sur les pastorales amène cependant les chercheurs à réduire les analyses au passé et au présent en évitant de pronostiquer l'avenir de telle ou telle fête.

\section{Historique du fonds}

La plupart des dons de la bibliothèque municipale de Bayonne datent de 1924, et plus précisément du 22 janvier et surtout du 21 mai.

\section{Bibliographie de Georges Hérelle}

Canico et Beltchitine. Farce charivarique. Traduite pour la première fois en français d'après le manuscrit unique de la Bibliothèque de Bordeaux et accompagnée d'une notice sur le théâtre basque et d'un commentaire. Paris, 1908, Editions Daragon.

"Les représentations de pastorales basques dans la Soule pendant la période révolutionnaire" in RIEV, IV, pp 5-17, 1910.

"Noticias curiosas acerca de las Pastorales", Euskalerriaren Alde, année 1, n7 et n8, pp 199204, 1911.

"Les charivaris nocturnes dans le Pays Basque français" in RIEV, XV, Donostia, pp 505-522, 1921.

"Les mascarades souletines", RIEV, pp 368-385, T VIII, 1914 et pp 159-190, T XIV, 1923.

Etudes sur le théâtre basque. La représentation des pastorales à sujets tragiques. Paris, Honoré Champion, 1923.

"Instruction pour la mise en scène des farces charivariques" in Gure Herria, pp 311-325, 1923.

Etudes sur le théâtre basque. Le théâtre comique. Paris, Honoré Champion, 1925.

Etudes sur le théâtre basque. Les pastorales à sujets tragiques considérées littérairement. Paris, Honoré Champion, 1926.

Etudes sur le théâtre basque. Le répertoire du théâtre tragique. Paris, Honoré Champion, 1928.

Les théâtres ruraux en France depuis le XIVème siècle jusqu'à nos jours. Paris, Honoré Champion, 1930.

Autres références concernant Georges Hérelle

Cavaillès, Henri, "Etudes. Georges Hérelle" in Bulletin du Musée Basque, n5, pp 375-386, 1933.

Fourcade, Auguste. - "Nécrologie. Georges Hérelle" in Bulletin de la Société des Sciences et Arts de Bayonne, pp 5-8, 1936. 
